\title{
Detailed dosimetric evaluation of intensity-modulated radiation therapy plans created for stage $C$ prostate cancer based on a planning protocol.
}

\section{AUTHOR(S):}

Norihisa, Yoshiki; Mizowaki, Takashi; Takayama, Kenji; Miyabe, Yuki; Matsugi, Kiyotomo; Matsuo, Yukinori; Narabayashi, Masaru; ... Nakamura, Akira; Nagata, Yasushi; Hiraoka, Masahiro

\section{CITATION:}

Norihisa, Yoshiki ...[et al]. Detailed dosimetric evaluation of intensity-modulated radiation therapy plans created for stage $C$ prostate cancer based on a planning protocol.. International journal of clinical oncology 2012, 17(5): 505-511

\section{ISSUE DATE:}

2012-10

URL:

http://hdl.handle.net/2433/165054

\section{RIGHT:}

The final publication is available at www.springerlink.com; This is not the published version. Please cite only the published version.; この論文 は出版社版でありません。引用の際には出版社版をご確認ご利用くだ さい。 


\section{Detailed dosimetric evaluation of intensity-modulated radiation therapy plans created for stage $C$ prostate cancer based on a planning protocol.}

Yoshiki NORIHISA ${ }^{1}$

Takashi MIZOWAKI ${ }^{1}$

Kenji TAKAYAMA ${ }^{1}$

Yuki MIYABE ${ }^{1}$

Kiyotomo MATSUGI ${ }^{1}$

Yukinori MATSUO ${ }^{1}$

Masaru NARABAYASHI ${ }^{1}$

Katsuyuki SAKANAKA ${ }^{1}$

Akira NAKAMURA ${ }^{1}$

Yasushi NAGATA ${ }^{2}$

Masahiro HIRAOKA ${ }^{1}$

${ }^{1}$ Department of Radiation Oncology and Image-applied Therapy, Graduate School of Medicine, Kyoto University, 54 Shogoin Kawahara-cho, Sakyo-ku, Kyoto 606-8507, Japan.

${ }^{2}$ Department of Radiation Oncology, Graduate School of Biomedical Sciences, Hiroshima University, 1-2-3 Kasumi, Minami-ku, Hiroshima 734-8551, Japan.

Corresponding author: Takashi Mizowaki, MD, $\mathrm{PhD}$,

Mailing address: Department of Radiation Oncology and Image-applied Therapy, Kyoto University

Graduate School of Medicine, 54 Shogoin Kawahara-cho, Sakyo-ku, Kyoto, 606-8507, Japan

Phone: +81-75-751-3419

Fax: $\quad+81-75-751-3422$

Email: mizo@kuhp.kyoto-u.ac.jp 


\section{ABSTRACT}

Background: Intensity-modulated radiation therapy (IMRT) has been employed as a precision radiation therapy with higher conformity to the target. Although clinical outcomes have been reported for many investigations, detailed treatment planning results have not been mentioned so far. The aim of this study was to evaluate the dose specifications of our IMRT treatment plans for locally advanced prostate cancer.

Methods: Seventy-seven clinically applied IMRT plans treated between September 2003 and December 2005, in which patients were irradiated 78Gy in the prone position, were retrospectively analyzed. Dosimetric data outputted from dose volume histograms were evaluated in detail.

Results: The mean dose \pm standard deviation, homogeneity index and conformity index to the planning target volume (PTV) were $78.3 \pm 0.7$ Gy $(100.4 \pm 0.9 \%), 13.7 \pm 3.0$, and $0.83 \pm$ 0.04 , respectively. For the clinical target volume, the mean dose was $80.3 \pm 0.7 \mathrm{~Gy}(102.9 \pm$ $0.9 \%$ ). The V40Gy, V60Gy, and V70Gy of the rectal wall were $58.3 \pm 2.8,29.6 \pm 2.7$, and $15.2 \pm 3.0 \%$, respectively. Planning difficulties were encountered in patients whose bowels were displaced downward, as constraints imposed by the bowel position altered the dose index of the PTV. In many cases, additional bowel optimization parameters were required to satisfy constraints for organs at risk. However, major deviation could be avoided by inverse planning with computer optimization.

Conclusion: IMRT allowed to create acceptable and practical treatment plans for locally advanced prostate cancer. Reports regarding detailed dosimetric evaluations are mandatory to interpret clinical outcomes in the future.

\section{Mini Abstract}

Detailed dosimetric data on IMRT plans for locally advanced prostate cancer were reported, which is mandatory for interpretation of clinical outcomes as well as creating planning protocols in the future.

Key words: prostate cancer, intensity-modulated radiation therapy (IMRT), radiation therapy treatment planning 


\section{INTRODUCTION}

Intensity-modulated radiation therapy (IMRT) has been employed as a precision radiation therapy offering higher conformity to the target ${ }^{1}$. IMRT can create complicated dose distributions achieving a dose escalation to the target volume and dose reduction of organs at risks (OAR) adjacent to the target using a computer optimization. Many clinical investigations were reported dosimetric and clinical advantages of IMRT for prostate cancers $^{2-7}$, head-and-neck tumors ${ }^{8,9}$, brain tumors ${ }^{10-12}$, gynecologic tumors ${ }^{13-15}$ and so forth.

Especially in the prostate cancer, its advantage is distinctive and widely applied as a routine clinical practice, and better clinical outcomes had been reported. However, details of treatment planning results were not mentioned in existing articles, which will make it difficult to compare outcomes among different institutions and to derive an optimal treatment planning policy from planning-outcome comparison studies. Because IMRT plans have essentially inhomogeneous dose distribution, it is very difficult to grasp the big picture of the treatment plans of IMRT based on some representative dosimetric index values alone such as D95. Therefore, this study was aimed to reveal the dose specifications of our routine IMRT planning protocol for locally advanced prostate cancer as well as to compare to our previous 3D-CRT methods ${ }^{16}$.

\section{MATERIALS AND METHODS}

\section{Patient characteristics}

Between September 2003 and December 2005, 85 patients with T3N0M0 prostate cancer, classified based on the 2002 definition of the International Union against Cancer, were consecutively treated by IMRT at our institution. Of those, eight cases were excluded from the analysis because of the following reasons. In five of the patients, the prescribed dose was reduced from 78 to 66-74 Gy due to diabetes mellitus, previous irradiation adjacent to the prostate, or a history of surgical management of rectal cancer; two other patients were irradiated using a setup position or port arrangement different from the routine protocol, and one patient had a huge atonic neurogenic urinary bladder and required a different planning concept to the bladder. Therefore, the present study was finally conducted on remaining 77 patients with T3N0M0 prostate cancer treated by standard IMRT plans. These patients were 
treated in the prone position and delivered $78 \mathrm{~Gy}$ to the target. Their characteristics are indicated in Table 1.

IMRT Planning protocols

Patients were immobilized in the prone position using a thermoplastic shell (Hip Fix system; Med-Tec Inc., Orange City, IA, USA), which extended from mid-thigh to the upper third of the leg in combination with a vacuum pillow (Vac-Lok system; Med-Tec Inc.) and a leg support. Planning computed tomography (CT) images were acquired using a CT simulator (CTS-20; Shimadzu, Kyoto, Japan) with a 5-mm slice thickness. Patients were instructed to void the urinary bladder and rectum about $1-1.5 \mathrm{~h}$ before the CT scan according to the patient's particular condition. Setup error was evaluated based on bony structures with liniacography (LG). Offline systematic error correction was performed with sequential five LG obtained in the first five irradiation, and weekly LG was taken for a setup verification.

All plans were carried out using 15-MV photon beams delivered by a Clinac 2300 C/D (Varian Medical Systems, Palo Alto, CA, USA) with a 40-leaf pair of multileaf collimators of 1-cm thickness. Treatment plans were created using an Eclipse-Helios system (Ver. 7; Varian Medical Systems, Palo Alto, CA, USA). The final dose distributions were calculated using a pencil beam convolution algorithm with a $5 \mathrm{~mm}$ by $5 \mathrm{~mm}$ calculation grid size. The modified Batho was applied for heterogeneity correction.

The prostate, seminal vesicles, rectal outer wall, bladder outer wall, small bowel, and large bowel were contoured. A clinical target volume (CTV) was created based on the prostate and seminal vesicles, which were contoured with reference to magnetic resonance images in most of the patients. The proximal two-thirds of the seminal vesicles were included in the CTV for T3a cases, and the entire seminal vesicles were included in the CTV for T3b patients. Inclusion of the seminal vesicles in the CTV resulted in larger CTVs in the current series than in the IMRT protocol used in a previous study, in which only the prostate was irradiated $^{16}$. Margins for the planning target volume (PTV) were added to the CTV according to the following 3D setting: 9-mm margins universally, except for a 6-mm margin posteriorly (for the direction to the rectum) and $10 \mathrm{~mm}$ superiorly (for the caudal direction). The rectal wall (RECT_W) was generated using the extract wall function of the treatment planning 
system (TPS) applying a 4-mm thickness. The existing RECT_W was uniform, ranging from $10 \mathrm{~mm}$ below the apex of the prostate to $10 \mathrm{~mm}$ above the tips of the seminal vesicles. The bladder wall (BLAD_W) was generated in the same manner with a 4-mm thickness. The PTV was divided into two components, with and without rectal overlap, for use in computer optimization. When the overlapping area of the PTV and bowels was large and caused difficulties in optimization, the PTV was further divided.

A five-field dynamic multileaf collimator technique was used for IMRT beam delivery. Gantry angles were $0,75,135,225$, and 285 degrees. Inverse treatment planning by computer optimization was conducted with the Helios system. The inverse optimizations were performed with the aim of fulfilling the planning goals establish in our planning protocol. The treatment planning goals to be achieved in the final dose distribution are listed in Table 2. Not only these hard constraints but also the following soft constrains were taken into account in accepting the final dose distribution, after fine adjustment of a normalization value. (1) The anterior rectal wall should receive $90-100 \%$ of the prescribed dose; the $95 \%$ isodose line usually lies between the anterior border of the rectal wall and the posterior border of the PTV. (2) There should be no significant hot spots outside the PTV. (3) The 50\% isodose line of the prescribed dose should generally not exceed the posterior wall of the rectum. These same constraints were also applied in the previous 3D-CRT plan used in our institution, in which patients were irradiated with $74 \mathrm{~Gy}$. When the plan did not match the OAR constraints despite adjustments of the optimization parameters, PTV dose coverage was sacrificed to give higher priority to the OARs.

Analyses of dose statistics

Planning data were analyzed using the outputs from the dose-volume histograms (DVH) generated by the TPS. D95 (D99) was defined as the percentage of the prescribed dose covering $95 \%(99 \%)$ of the volume. V90 was the percentage volume that received at least $90 \%$ of the prescribed dose. V40Gy was defined as the percentage volume that received at least 40 Gy. The dose inhomogeneity of the PTV was defined using the following equation: Homogeneity index $(\mathrm{HI})=(\mathrm{D} 5-\mathrm{D} 95) /$ mean dose $\times 100$. Dose conformity to the PTV was calculated as the conformity index (CI) advocated by van't Riet ${ }^{17}$ : CI = 
$6 / 16$

$\left(\mathrm{V} 95_{\mathrm{PTV}} / \mathrm{V}_{\mathrm{PTV}}\right) \times\left(\mathrm{V} 95_{\mathrm{PTV}} / \mathrm{V} 95_{\mathrm{BODY}}\right)$. Here, V95 PTV $_{\text {is }}$ the V95 of the PTV, $\mathrm{V}_{\mathrm{PTV}}$ is the volume of the PTV, and V95

The patients were divided into two groups based on anatomic conditions. Group B- consisted of patients who did not need optimization constraints for the bowel, because either no or only a very small volume of bowel was included in the irradiated field. Group B+ comprised patients who required additional bowel optimization parameters to satisfy the final dose constraints. Statistical calculations were performed with PRISM 4 software (GraphPad Software, San Diego, CA, USA).

\section{RESULTS}

Treatment planning

A typical dose distribution in the trans-axial plane is shown in Figure 1. Figure 2 illustrates the DVH, in which the mean and standard deviations of the PTV, RECT_W, and BLAD_W are indicated. Target doses statistics are summarized in Table 3. The mean dose (mean \pm standard deviation) to the PTV was $78.3 \pm 0.7$ Gy $(100.4 \pm 0.9 \%$ ), with a maximum dose of $84.7 \pm 0.8$ Gy $(108.6 \pm 1.0 \%)$. The V90, D95, and D99 were 97.3 $\pm 2.5,92.4 \pm 2.9$, and $86.7 \pm 5.6 \%$, respectively. The HI was $13.7 \pm 3.0$, and the CI was $0.83 \pm 0.04$. For the CTV, the mean dose was $80.3 \pm 0.7$ Gy $(102.9 \pm 0.9 \%)$, and the V90, D95, and D99 were $99.9 \pm 0.4,98.7 \pm 1.2$, and $97.0 \pm 2.3 \%$, respectively.

Plan normalization values were adjusted to meet the dose constraints for the OAR. The doses to the RECT_W and BLAD_W are summarized in Table 4. The V40Gy, V60Gy, and V70Gy of the RECT_W were $58.3 \pm 2.8,29.6 \pm 2.7$, and $15.2 \pm 3.0 \%$, respectively. The V40Gy and V70Gy of the BLAD_W were $41.6 \pm 10.8$ and $20.6 \pm 5.1 \%$, respectively.

The V65Gy of the large bowel exceeded $0.50 \mathrm{ml}$ in 13 patients $(0.51-2.11 \mathrm{ml})$. However, V65Gy exceeded $1.0 \mathrm{ml}$ was observed in only one patient $(2.11 \mathrm{ml})$. The V60Gy of the small bowel $>0.50 \mathrm{ml}$ was observed in five patients $(0.51-1.07 \mathrm{ml})$, but reached $1.0 \mathrm{ml}$ in only one patient.

If the initial goals could not be satisfied, the optimization parameters were relaxed to meet the clinical requirements; to meet the rectal wall constraints, the PTV coverage was sacrificed. While treatment planning goals for the OAR were achieved in most cases (Table 5), PTV 
coverage could not be preserved in several patients presenting with anatomical difficulties.

Impact of the bowels on target coverage

Difficulties were encountered in treatment planning for patients whose bowels were displaced downward. Figure 3 indicates the differences in the doses administered to the target volumes between the two groups. Forty-eight patients were classified to the group B+, where additional optimization parameters for the bowels were needed, while 29 cases were assigned to the group B-. For the PTV, the D95, V90, and D99 in group B- versus group B+ were $93.7 \pm 1.2$ vs. $91.5 \pm 3.3 \%(p=0.0006), 98.6 \pm 1.1$ vs. $96.6 \pm 2.8 \%(p=0.0003)$, and $89.6 \pm 2.2$ vs. $84.9 \pm 6.3 \%(p<0.0001)$, respectively. The values in the group B- were significantly higher than those in the group B + . Significant differences in $\mathrm{HI}$ and CI were also observed. On the other hand, there was no significant difference in the mean dose of the PTV and CTV, or in the D95 of the CTV between the groups.

\section{DISCUSSION}

Intensity-modulated radiation therapy (IMRT) has been employed as a precision radiation therapy with higher conformity to the target. Although clinical outcomes have been reported for many investigations, results of treatment planning have not mentioned in detail so far. Therefore, the aim of this study was to evaluate the dose specifications of our IMRT treatment plans for locally advanced prostate cancer.

In an Australian study reported by Skala et $a l^{18}$, three institutions created IMRT plans for their patients with prostate cancer using the same contours and dose constraints. While the final dose constraints could be satisfied without great difficulty, there were large differences in the DVH curves of the OAR. However, determination of inter-institutional homogeneity was incomplete due to a lack of detailed information regarding the planning concept. In the present study, clinical plans were designed at a single institute by three radiation oncologists and five medical physics personnel. Homogeneous plans could be achieved within the defined soft and hard constraints.

The process of IMRT treatment planning, in which the dose distribution is adjusted to achieve a balance between targets and OARs, differs from that of conventional 


\section{$8 / 16$}

radiotherapy planning. In IMRT plans, the dose distribution is in essentials not homogeneous; this is not the case for 3D-CRT plans, which are difficult to adapt because they consist solely of a prescribed dose determined using simple methods that have been universally adopted in conventional radiotherapy, e.g., the point dose to the isocenter. Discrepancies between the "official" prescribed dose of IMRT plans and that of 3D-CRT plans occur not only in the inter-institutional variation but also in the intra-institutional variation. The PTV mean doses of IMRT plans in the present study (CTV $=$ prostate + whole or $2 / 3$ of seminal vesicles $)$ and 3D-CRT plans in a previous analysis ( CTV $=$ prostate alone ) were $100.4 \pm 0.9$ and $99.5 \pm$ $0.3 \%$, respectively, while the corresponding CTV values were $102.9 \pm 0.9$ and $100.7 \pm 0.7 \%$, respectively ${ }^{16}$

Unlike the conventional radiation treatment planning with the dose prescription to the isocenter, the final dose distribution of IMRT has larger variation because inverse planning deviates from the optimization constraints; that is, the dosimetric values determined by the planner (e.g., prescribed dose) is one of several optimization parameters. Normalization with the D95 of the PTV overlapping the OAR may cause large differences in the PTV mean dose, depending on the volume ratio included in the OAR, and may increase treatment inhomogeneity. In contrast, the application of multiple dose constraints as applied in our protocol results in rather homogeneous mean doses to the target. In designing our planning protocol for prostate IMRT, we decided to give higher priority to avoid potential dose escalation and larger variation in the mean dose to the target caused by the D95 dose prescription. Therefore, our $78 \mathrm{~Gy}$ plan was defined as plans satisfying the previously-described soft and hard constraints, which resulted in very small variations in the mean doses to both the CTV and the PTV as indicated in Table 3. In our planning protocol, the coverage, homogeneity, and conformity were sacrificed to spare the bowels, while the CTV dose was maintained. In other words, the PTV dose was decreased to provide adequate clinical optimization. IMRT plans often require this kind of compromise to avoid extreme deviations.

In this study, rectal radiation doses were analyzed based on relative volumes, using software designed for the TPS. Munbodh et al. analyzed the radiation dose on the rectum surface to identify indicators of late rectal toxicity ${ }^{19}$. However, this approach poses several 


\section{$9 / 16$}

clinical difficulties such as necessity of the special in-house software. In contrast, evaluating the dose to the OAR based on the absolute volume appears to result in less inter-institutional deviation. Thus, details of rectal DVH analyses based on a uniform delineation and absolute volumes may serve as a useful alternative indicator. In the meantime, it is important to create standard DVH models and make them available for clinical evaluation.

Several guidelines or recommendations for documenting and reporting IMRT plans have been published ${ }^{20,21}$. After achieving homogeneity in terms of target delineation and optimization, detailed reporting and analysis are indispensable in the interpretation and comparison of clinical outcomes ${ }^{21}$. This is also true within a single institute, where a thorough review of treatment planning results is essential to evaluate clinical outcome. The precise and quantitative evaluation of treatment plans may contribute to the development of new treatment protocols or guidelines in the future.

Recently, several image-guided radiotherapy (IGRT) techniques have introduced in IMRT for prostate cancer. Prostate-based online error correction can be performed using fiducial marker, $\mathrm{CT}$ on rails, cone beam $\mathrm{CT}$, ultrasound or electric magnetic signals. This article was based on rather early IMRT cases for prostate cancers in our institute, setup error correction was performed with offline method, the PTV margin is comparatively not so small. Decreased CTV to PTV margin is expected to toxicity reduction, and have make possible higher dose delivery to prostate. It's clinical impact have not established yet, clinical application requires careful consideration to avoid decreased tumor control ${ }^{22}$.

In conclusion, planning outcomes of a clinical IMRT planning protocol for prostate cancer were evaluated. Several dose constraints for PTV and OAR were sufficient to maintain planning homogeneity. In patients with downward displacement of the small and large bowels, additional optimization parameters were often required. Although this sacrificed PTV coverage, major deviations from the constraints were avoided due to computer optimization. The precise evaluation of clinical plans, as conducted in the present study, is indispensable to understanding the inter- and intra-institutional differences in clinical outcomes. This analysis provides an important basis upon which to design treatment planning protocols as well as interpreting clinical outcomes of the current protocol in the future. 


\section{$10 / 16$}

\section{CONFLICT OF INTERST STATEMENT}

None of the authors have any financial relationship with any commercial entity that has an interest in the subject matter or materials discussed in this manuscript.

\section{ACKNOWLEGEMENTS}

This article was partly supported by Grant-in-aid for research on radiation oncology of JASTRO, 2009-2010.

\section{FIGURES}

Fig. 1 Typical dose distributions in the trans-axial (a) and sagittal (b) plane.

(a)

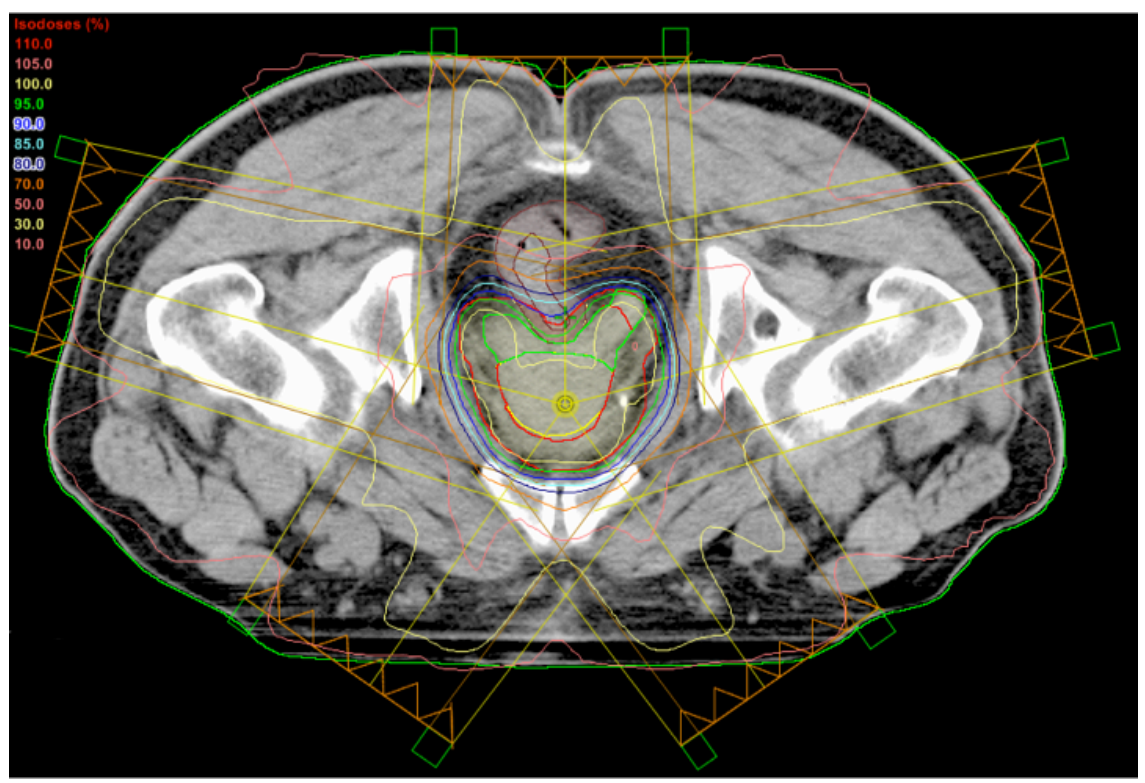

(b)

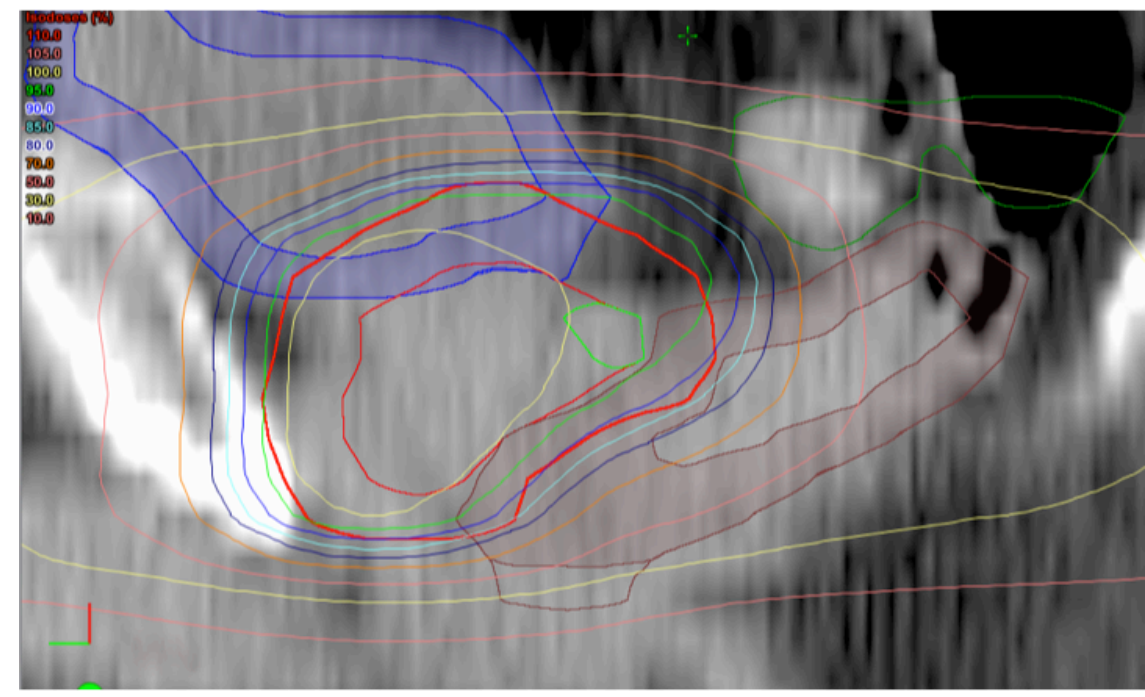




\section{$11 / 16$}

Fig. 2

Dose-volume histograms (DVHs) indicating mean and standard deviation of the (a) planning target volume (PTV), (b) rectal wall (RECT_W), and (c) bladder wall (BLAD_W).

(a) PTV

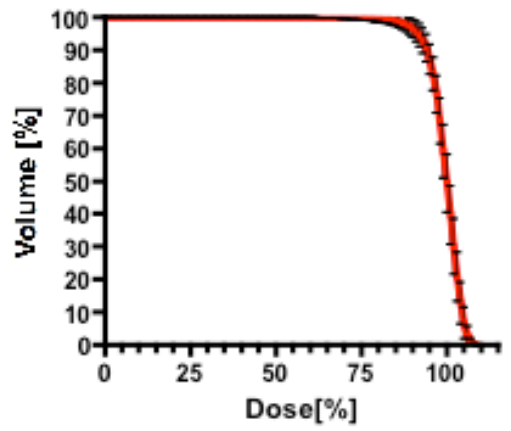

(b) RECT_W

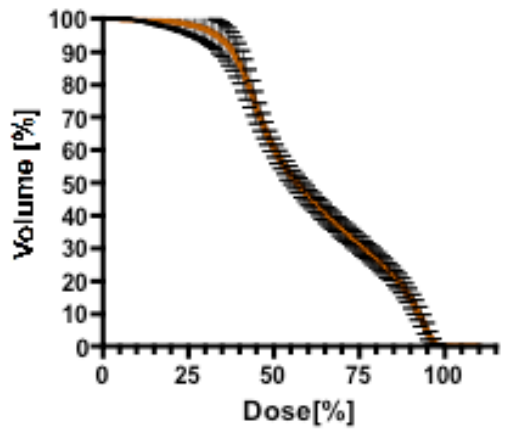

(c) BLAD_W

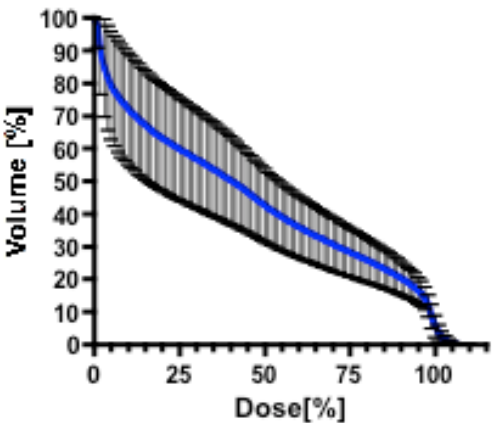

Fig. 3

The differences of index values in the doses administered to the target volumes. IMRT plans were stratified whether additional optimization parameters for the bowels were needed (group $\mathrm{B}+, \mathrm{n}=48$ ) or not (group B-, n = 29).

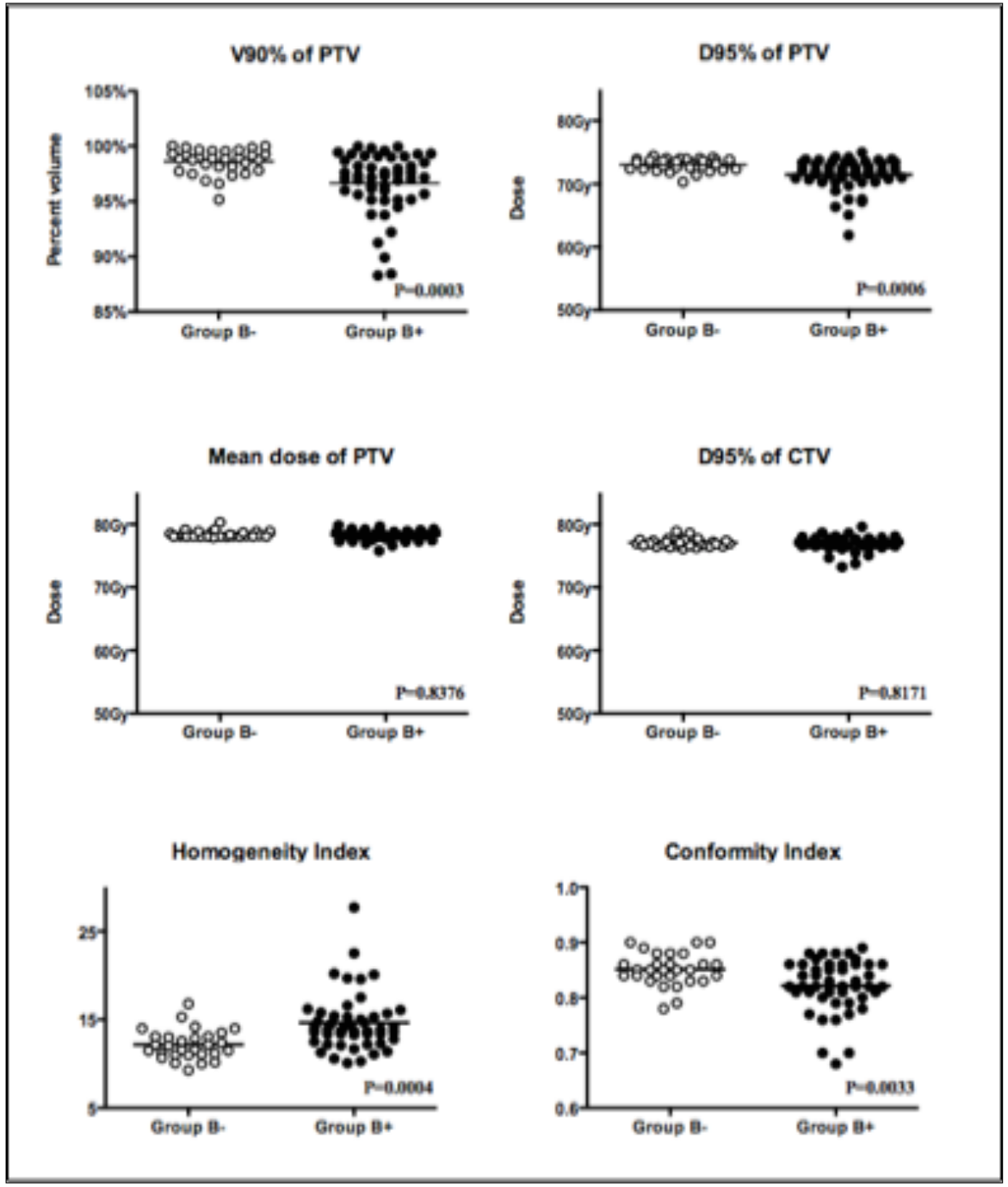


$12 / 16$

\section{TABLES}

Table 1. Patient characteristics

\begin{tabular}{|c|c|c|}
\hline \multicolumn{3}{|l|}{ Clinical Stage } \\
\hline \multicolumn{3}{|l|}{ T3aN0M0 : $\mathrm{n}=52$} \\
\hline \multicolumn{3}{|l|}{ T3bN0M0 : $n=25$} \\
\hline & median & range \\
\hline Age & 72 & $(56-80)$ \\
\hline Prostate volume $(\mathrm{ml})$ & 27.4 & $(13.7-52.8)$ \\
\hline CTV volume ( $\mathrm{ml}$ ) & 37.7 & $(20.6-64.6)$ \\
\hline PTV volume ( $\mathrm{ml})$ & 120.9 & $(73.0-176.5)$ \\
\hline \multirow{2}{*}{$\begin{array}{l}\text { Rectal wall volume }(\mathrm{ml}) \\
\text { Bladder wall volume } \\
(\mathrm{ml})\end{array}$} & 36.2 & $(21.2-72.4)$ \\
\hline & 78.9 & $(35.4-145.7)$ \\
\hline Large bowel ${ }^{*}(\mathrm{ml})$ & 13.0 & $(0.0-94.1)$ \\
\hline Small bowel ${ }^{*}(\mathrm{ml})$ & 0.0 & $(0.0-88.2)$ \\
\hline
\end{tabular}

*the volume involved in radiation field

Table 2. Summary of planning goals

\begin{tabular}{lll}
\hline Structure & & Constraint (preferable) \\
\hline PTV & D95 & $\geq 90 \%(\geq 95 \%)$ \\
& V90 & $\geq 96 \%(\geq 98 \%)$ \\
& Max & $\leq 110 \%$ \\
RECT_W & V40Gy & $\leq 65 \%(\leq 60 \%)$ \\
& V60Gy & $\leq 35 \%(\leq 30 \%)$ \\
& V70Gy & $\leq 25 \%(\leq 20 \%)$ \\
& V78Gy & $<1 \%$ \\
BLAD_W & V40Gy & $\leq 65 \%(\leq 60 \%)$ \\
& V70Gy & $\leq 35 \%$ \\
Bowel_L & V65Gy & $\leq 1.0 \mathrm{ml}(\leq 0.5 \mathrm{ml})$ \\
Bowel_S & V60Gy & $\leq 1.0 \mathrm{ml}(\leq 0.5 \mathrm{ml})$ \\
\hline
\end{tabular}

PTV: planning target volume, RECT_W: rectal wall, BLAD_W: bladder wall, Bowel_S: small bowel, Bowel_L: large bowel 
$13 / 16$

Table 3. Results of radiation treatment planning for targets

\begin{tabular}{llll}
\hline & Prostate & CTV & PTV \\
\hline V90 (\%) & $100.0 \pm 0.0$ & $99.9 \pm 0.4$ & $97.3 \pm 2.5$ \\
D95 (\%) & $99.2 \pm 0.9$ & $98.7 \pm 1.2$ & $92.4 \pm 2.9$ \\
D99 (\%) & $97.8 \pm 0.9$ & $97.0 \pm 2.3$ & $86.7 \pm 5.6$ \\
Minimum dose (Gy) & $74.8 \pm 1.2$ & $73.2 \pm 3.1$ & $60.5 \pm 6.3$ \\
Maximum dose (Gy) & $84.5 \pm 0.9$ & $84.7 \pm 0.9$ & $84.7 \pm 0.8$ \\
Mean dose (Gy) & $80.6 \pm 0.7$ & $80.3 \pm 0.7$ & $78.3 \pm 0.7$ \\
Homogeneity index & - & - & $13.7 \pm 3.0$ \\
Conformity index & - & - & $0.83 \pm 0.04$ \\
\hline
\end{tabular}

Values are mean \pm SD.

Table 4. Results of radiation treatment planning ( OARs )

\begin{tabular}{lll}
\hline & Rectal wall & Bladder wall \\
\hline V40Gy (\%) & $58.3 \pm 2.8$ & $41.6 \pm 10.8$ \\
V60Gy (\%) & $29.6 \pm 2.7$ & $27.4 \pm 7.2$ \\
V70Gy (\%) & $15.2 \pm 3.0$ & $20.6 \pm 5.1$ \\
\hline
\end{tabular}

Values are mean \pm SD.

Table 5. Achievement ratio of treatment planning goals.

\begin{tabular}{llll}
\hline Structure & & Achievement of constraint & Achievement of preferable \\
\hline PTV & D95 & $90 \%$ & $22 \%$ \\
& V90 & $83 \%$ & $58 \%$ \\
& Max & $99 \%$ & - \\
RECT_W & V40Gy & $100 \%$ & $84 \%$ \\
& V60Gy & $100 \%$ & $56 \%$ \\
& V70Gy & $100 \%$ & $96 \%$ \\
& V78Gy & $100 \%$ & - \\
BLAD_W & V40Gy & $100 \%$ & $97 \%$ \\
& V70Gy & $100 \%$ & - \\
Bowel_L & V65Gy & $99 \%$ & $91 \%$ \\
Bowel_S & V60Gy & $99 \%$ & $94 \%$ \\
\hline
\end{tabular}

PTV: planning target volume, RECT_W: rectal wall, BLAD_W: bladder wall, Bowel_S: small bowel, Bowel_L: large bowel 
$14 / 16$

\section{REFERENCES}

1. Galvin JM, Ezzell G, Eisbrauch A, et al. (2004) Implementing IMRT in clinical practice: a joint document of the American Society for Therapeutic Radiology and Oncology and the American Association of Physicists in Medicine. Int J Radiat Oncol Biol Phys 58:1616-1634

2. Pollack A, Zagars GK, Rosen, II (1999) Prostate cancer treatment with radiotherapy: maturing methods that minimize morbidity. Semin Oncol 26:150-161

3. Teh BS, Mai WY, Augspurger ME, et al. (2001) Intensity modulated radiation therapy (IMRT) following prostatectomy: more favorable acute genitourinary toxicity profile compared to primary IMRT for prostate cancer. Int J Radiat Oncol Biol Phys 49:465-472

4. Storey MR, Pollack A, Zagars G, et al. (2000) Complications from radiotherapy dose escalation in prostate cancer: preliminary results of a randomized trial. Int J Radiat Oncol Biol Phys 48:635-642

5. Zelefsky MJ, Fuks Z, Happersett L, et al. (2000) Clinical experience with intensity modulated radiation therapy (IMRT) in prostate cancer. Radiother Oncol 55:241-249

6. Zelefsky MJ, Fuks Z, Hunt M, et al. (2001) High dose radiation delivered by intensity modulated conformal radiotherapy improves the outcome of localized prostate cancer. J Urol 166:876-881

7. Pollack A, Zagars GK, Starkschall G, et al. (2002) Prostate cancer radiation dose response: results of the M. D. Anderson phase III randomized trial. Int J Radiat Oncol Biol Phys 53:1097-1105

8. Kam MK, Leung SF, Zee B, et al. (2007) Prospective randomized study of intensity-modulated radiotherapy on salivary gland function in early-stage nasopharyngeal carcinoma patients. J Clin Oncol 25:4873-4879

9. Teo PM, Ma BB, Chan AT (2004) Radiotherapy for nasopharyngeal carcinoma--transition from two-dimensional to three-dimensional methods. Radiother Oncol 73:163-172

10. Roberge D, Kun LE, Freeman CR (2005) Intracranial germinoma: on whole-ventricular irradiation. Pediatr Blood Cancer 44:358-362 
11. Iuchi T, Hatano K, Narita Y, et al. (2006) Hypofractionated high-dose irradiation for the treatment of malignant astrocytomas using simultaneous integrated boost technique by IMRT. Int J Radiat Oncol Biol Phys 64:1317-1324

12. Narayana A, Yamada J, Berry S, et al. (2006) Intensity-modulated radiotherapy in high-grade gliomas: clinical and dosimetric results. Int J Radiat Oncol Biol Phys 64:892-897

13. Roeske JC, Lujan A, Rotmensch J, et al. (2000) Intensity-modulated whole pelvic radiation therapy in patients with gynecologic malignancies. Int J Radiat Oncol Biol Phys 48:1613-1621

14. Beriwal S, Gan GN, Heron DE, et al. (2007) Early clinical outcome with concurrent chemotherapy and extended-field, intensity-modulated radiotherapy for cervical cancer. Int J Radiat Oncol Biol Phys 68:166-171

15. Mundt AJ, Lujan AE, Rotmensch J, et al. (2002) Intensity-modulated whole pelvic radiotherapy in women with gynecologic malignancies. Int J Radiat Oncol Biol Phys 52:1330-1337

16. Zhu S, Mizowaki T, Nagata Y, et al. (2005) Comparison of three radiotherapy treatment planning protocols of definitive external-beam radiation for localized prostate cancer. Int J Clin Oncol 10:398-404

17. van't Riet A, Mak AC, Moerland MA, et al. (1997) A conformation number to quantify the degree of conformality in brachytherapy and external beam irradiation: application to the prostate. Int J Radiat Oncol Biol Phys 37:731-736

18. Skala M, Holloway L, Bailey M, et al. (2005) Australia-wide comparison of intensity modulated radiation therapy prostate plans. Australas Radiol 49:222-229

19. Munbodh R, Jackson A, Bauer J, et al. (2008) Dosimetric and anatomic indicators of late rectal toxicity after high-dose intensity modulated radiation therapy for prostate cancer. Med Phys 35:2137-2150

20. Holmes T, Das R, Low D, et al. (2009) American Society of Radiation Oncology recommendations for documenting intensity-modulated radiation therapy treatments. Int J Radiat Oncol Biol Phys 74:1311-1318

21. Das IJ, Cheng CW, Chopra KL, et al. (2008) Intensity-modulated radiation therapy 
$16 / 16$

dose prescription, recording, and delivery: patterns of variability among institutions and treatment planning systems. J Natl Cancer Inst 100:300-307

22. Engels B, Soete G, Verellen D, et al. (2009) Conformal arc radiotherapy for prostate cancer: increased biochemical failure in patients with distended rectum on the planning computed tomogram despite image guidance by implanted markers. Int $\mathbf{J}$ Radiat Oncol Biol Phys 74:388-391 